DOI: 10.17707/AgricultForest.62.4.16

Halil BOLU ${ }^{1}$

\title{
SOUTHEASTERN ANATOLIA REGION INSECT FAUNA I (COLEOPTERA I: CARABOIDEA; DYTISCOIDEA; BOSTRICHOIDEA; CHRYSOMELOIDEA; CLEROIDEA; CUCUJOIDEA) OF TURKEY
}

\section{SUMMARY}

The aim of this study was to determine Insect fauna Southeastern Anatolia Region. Surveys on insect species in various ecologies have been conducted in the provinces (Adıyaman, Batman, Gaziantep, Diyarbakır, Mardin, Siirt, Şanlıurfa, Şırnak) of Southeastern Anatolia region between the years 1948-2013. Almost 2600 species and subspecies among 180 families belonging to 13 insect orders are defined owing to these studies. Coleoptera species formed about $20 \%$ of the collected insects. Coleoptera included 32 families were recorded. During this study totally 268 species were found in 13 families and in 6 superfamilies of Coleoptera. Those superfamilies are Caraboidea, Dytiscoidea, Bostrichoidea, Chrysomeloidea, Cleroidea and Cucujoidea. The distribution of determined insect types according to the provinces, plant hosting and feeding type is also done. Information about their identification, host plants, and distribution in Southeastern Anatolia Region was presented as detailed. \section{Turkey. \\ Keywords: Southeastern Anatolia Region, Insect Fauna, Coleoptera,}

\section{INTRODUCTION}

Insects (Insecta) are the most numerous group of animals in the world, with over one million species that have been described (Price, 1997). Insects are difficult to study because they represent the most species-rich, yet one of the least known, of all taxa of living organisms, a problem that is compounded by a dearth of skilled entomologists. Although the number of described insect species is uncertain due to synonyms and the lack of a global list, most authorities recognize 900000-1000000 named morpho-species, representing 56\% of all species known on Earth (Groombridge, 1992; Anonymous, 2003). Sensible estimates of the number of insects yet to be discovered range from another 1 million to 30 million species (Erwin, 1982; 1991), although most predict around 2-8 million more species (May, 1990; Gaston, 1991; Stork, 1997; Ødegaard, 2000). Conservative estimates suggest that $50-90 \%$ of the existing insect species on Earth have still to be discovered, yet the named insects alone comprise more than half of all known species of organism.

Insects constitute the most diverse form of animal life in terrestrial ecosystems. Most species are innocuous and essential components of natural

\footnotetext{
${ }^{1}$ Halil Bolu, (corresponding author: besni@dicle.edu.tr) Department of Plant Protection, Faculty of Agriculture, Dicle University, 21280 Diyarbakır, TURKEY.

Notes: The authors declare that they have no conflicts of interest. Authorship Form signed online.
} 
ecosystems. Because they are cold-blooded, the rates of key physiological processes in their life cycles are determined by environmental conditions, especially temperature and precipitation. In general they have short generation times, high fecundity and high mobility (Moore and Allard, 2008). Turkey in fact seems to be like a small continent in terms of biological diversity. Despite the Anatolia is not a continent alone, it contains all properties of a continent that should have an ecosystem and habitat. Each of seven geographical regions in Turkey has a distinguishable climate, flora and fauna. The aim of this study is to make contribution to some faunistical records of Coleoptera of the Southeastern Anatolia Region of Turkey.

\section{MATERIAL AND METHODS}

Southeastern Anatolia Region (Adiyaman, Batman, Gaziantep, Diyarbakır, Mardin, Siirt, Şanlıurfa, Şırnak) entomology studies on insect species in different ecological provinces were made between the years 1948-2013 (Figure 1).

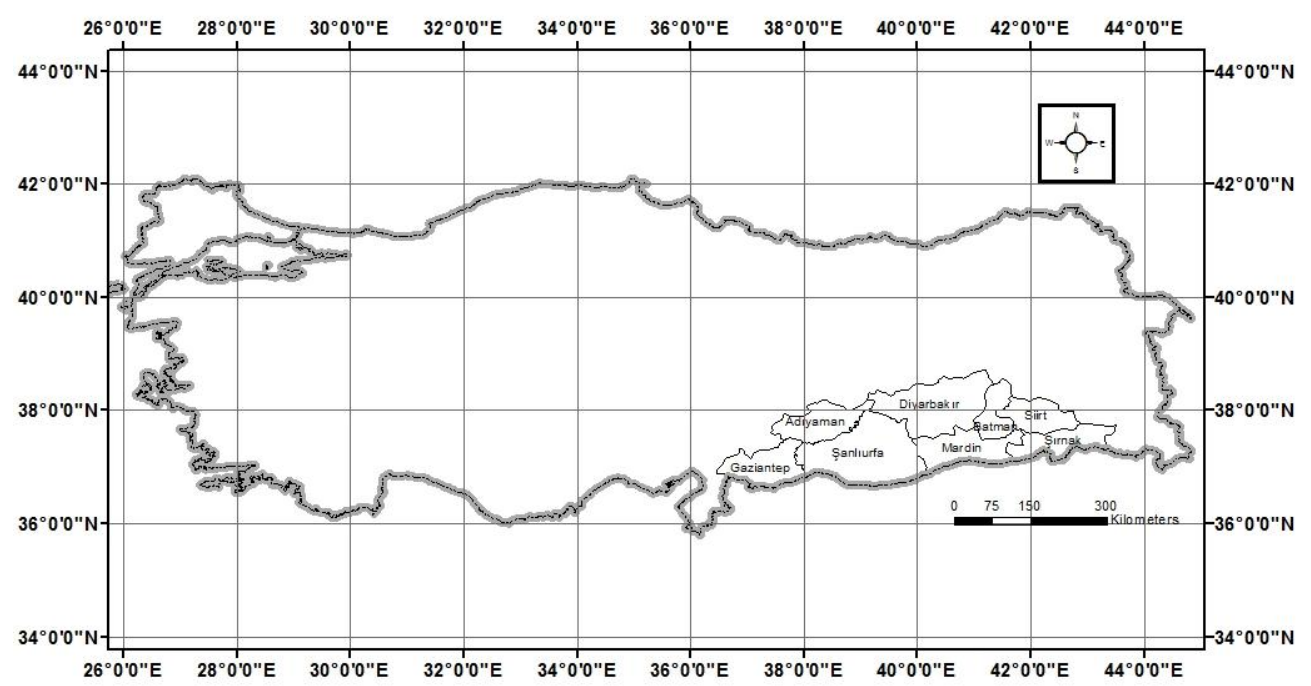

Figure 1. Sampling localities in Southeastern Anatolia Region of Turkey

In this study, we prepared for the inventory has reached the major advantage of the waterways:

-Currently in Turkey, published or unpublished entomology journals related to scanning,

-Giving more weight to faunistic studies, and in the meantime, the insect fauna of our country foreign scientific journals that publishes articles about scanning,

-Faculty of Agriculture, Faculty of Science and Regional Plant Protection Research Institute in the library of books on insect fauna and the screening of the booklet, 
-The doctor and the master's thesis of entomology in the region on the scanning,

-Review of other studies on the insect fauna in the area. obtained.

In this study, we evaluated the information as described above were

\section{RESULTS AND DISCUSSION}

Surveys on insect species in various ecologies have been conducted in the provinces (adiyaman, batman, gaziantep, diyarbakir, mardin, siirt, şanliurfa, şirnak) of southeastern anatolia region between the years 1948-2013. Almost 2600 species and subspecies almost 180 families belonging to 13 insect orders are defined owing to these studies. During this study totally 268 species were found in 13 families and in 6 superfamilies of coleoptera. Those superfamilies are caraboidea, dytiscoidea, bostrichoidea, chrysomeloidea, cleroidea and cucujoidea. The number and percentages of the species of the families are given on Table 1.

Table 1. Number of species of order Coleoptera on Southeastern Anatolia Region

\begin{tabular}{|c|c|c|c|}
\hline Superfamily & Family & Number Species & $\%$ \\
\hline Caraboidea & Carabidae & 18 & 6,71 \\
\hline & Cicindellidae & 6 & 2,23 \\
\hline Dytiscoidea & Dytiscidae & 1 & 0,37 \\
\hline Bostrichoidea & Bostrichidae & 3 & 1,12 \\
\hline & Dermestidae & 9 & 3,36 \\
\hline Chrysomeloidea & Cerambycidae & 60 & 22,39 \\
\hline & Chrysomelidae & 82 & 30,60 \\
\hline & Bruchidae & 17 & 6,34 \\
\hline Cleroidea & Trogossitidae & 1 & 0,37 \\
\hline Cucujoidea & Coccinellidae & 66 & 24,63 \\
\hline & Nitidulidae & 2 & 0,75 \\
\hline & Phalacridae & 2 & 0,75 \\
\hline & Silvanidae & 1 & 0,37 \\
\hline Total & 13 & 268 & 100,0 \\
\hline
\end{tabular}

The distribution of insect species in the Southeastern Anatolia Region as a result of studies carried out in different provinces ecology, information on the host status and feeding has been given below.

\section{Order Coleoptera (beetles) \\ Suborder Adephaga \\ Superfamily Caraboidea \\ Family Carabidae}

Amara aenea (De Geer, 1774), Distribution: Diyarbakır Host plant: Karacadağ mountain 
Amara (Amara) eurynota (Panzer, 1797), Distribution: Gaziantep Host plant: unknown

Chlaenius festivus (Panzer, 1796), Distribution: Southeastern Anatolia Region Host plant: unknown

Corsyra fusula (Fischer von Waldheim, 1820), Distribution: Diyarbakır, Mardin Host plant: Amygdalus communis L.

Daptus vittatus Fischer von Waldheim, 1823, Distribution: Diyarbak1r, Mardin Host plant: Amygdalus communis L.

Harpalus sp. nr.aeneus F., Distribution: Diyarbakır Host plant: Karacadağ mountain

Harpalus sp. nr.distinguendus, Distribution: Gaziantep Host plant: unknown

Harpalus (Harpalus) distinguendus distinguengus (Duftschmid, 1812), Distribution: Gaziantep Host plant: unknown

Harpalus (harpallophanus) haspesarmenus Schaubeger, 1926, Distribution: Adiyaman Host plant: unknown

Harpalus saxicola Dejean, 1829, Distribution: Southeastern Anatolia Region Host plant: unknown

Harpalus (s.str.) serripes (Quensel, 1806), Distribution: Adiyaman Host plant: unknown

Pseudoophonus rufipes (De Geer, 1774), Distribution: Diyarbakır Host plant: unknown

Parazuphium chevrolati (Castelnau, 1833), Distribution: Southeastern Anatolia Region Host plant: unknown

Zabrus blapoides Creutz, 1799, Distribution: Diyarbakır Host plant: unknown

Zabrus ruqulosus Kraatz, 1884, Distribution: Siirt Host plant: unknown

Zabrus sp. nr. socialis Sch., Distribution: Diyarbakır Host plant: Karacadağ mountain

Zabrus spinipes (Fabricius, 1798), Distribution: Batman, Diyarbakır, Siirt Host plant: unknown

Zabrus tenebrioides (Goeze, 1777), Distribution: Adiyaman, Diyarbakır Host plant: unknown

Family Cicindellidae

Cicindella campestris Linnaeus, 1758, Distribution: Diyarbakır Host plant: unknown

Cicindela herbacea Klug, 1832, Distribution: Adiyaman, Gaziantep Host plant: unknown

Calomera fischeri fischeri (Adams, 1817), Distribution: Adiyaman, Gaziantep, Siirt, Şanlıurfa Host plant: unknown

Homodela ismenia kilikiensis (Mandl, 1961), Distribution: Adıyaman, Gaziantep Host plant: unknown

Lophyridia fischeri (Adams, 1817), Distribution: Adiyaman, Gaziantep, Mardin, Siirt, Şanlıurfa Host plant: unknown 
Lophyridia littoralis (Mandl, 1967), Distribution: Mardin, Siirt, Şanlıurfa Host plant: unknown

\section{Superfamily Dytiscoidea}

Family Dytiscidae

Deronectes evelynae Fery and Hosseinie, 1998, Distribution: Adiyaman, Gaziantep Host plant: unknown

Suborder Polyphaga

Superfamily Bostrichoidea

Family Bostrichidae

Rhyzopertha dominica (Fabricius, 1792), Distribution: Adiyaman, Diyarbakır, Mardin, Siirt, Şanlıurfa Host plant: Triticum spp., Flour, Storage pest

Sinoxylon sexdentatum (Olivier, 1790), Distribution: Adiyaman, Batman, Mardin, Siirt, Şanlıurfa Host plant: Pistacia vera $\mathrm{L}$.

Stenomera assyrica Lesne, 1895, Distribution: Adıyaman, Batman, Mardin, Siirt, Şanlıurfa Host plant: Pistacia vera L.

\section{Family Dermestidae}

Attagenus (s.str.) orientalis Reitter in Schneider et Leder, 1878, Distribution: Mardin, Siirt, Şanlıurfa Host plant: Fruit, Pistacia vera L.

Attagenus (s.str.) quadrimaculatus Kraatz, 1858, Distribution: Gaziantep Host plant: unknown

Anthrenus (s.str.) delicatus Kiesenwetter, 1851, Distribution: Gaziantep Host plant: unknown

Anthrenus (s.str.) rotundulus rotundulus Reitter, 1889, Distribution: Gaziantep Host plant: unknown

Anthrenus (s.str.) simonis Reitter, 1881, Distribution: Diyarbakır Host plant: Light trap

Anthrenus (Nathrenus) pubifer Reitter, 1899, Distribution: Diyarbakır, Siirt Host plant: unknown

Anthrenus verbasci (Linnaeus, 1767), Distribution: Adiyaman, Batman, Diyarbakır, Gaziantep, Mardin, Siirt, Şanlıurfa, Şırnak Host plant: Amygdalus communis L., Cucurbita spp.

Trogoderma granarium Everts, 1898, Distribution: Adiyaman, Diyarbakır, Mardin, Siirt, Şanlıurfa Host plant: Amygdalus communis L., Storage pests, Cucurbita spp.

Trogoderma versicolor (Creutzer, 1799), Distribution: Diyarbakır Host plant: Zea mays L.

\section{Superfamily Chrysomeloidea}

Family Cerambycidae

Agapanthia dahli (Richter, 1821), Distribution: Gaziantep, Siirt Host plant: unknown 
Agapanthia cardui (Linnaeus, 1767), Distribution: Diyarbakır, Siirt Host plant: unknown

Agapanthia (s.str) lais Reiche \& Saulcy, 1858, Distribution: Mardin Host plant: unknown

Agapanthia kirbyi (Gyllenhal, 1817), Distribution: Siirt Host plant: unknown

Agapanthia walteri Reitter, 1898, Distribution: Mardin Host plant: unknown

Arhopalus syriacus (Reitter, 1895), Distribution: Gaziantep, Şanliurfa Host plant: unknown

Aromia moschata (Linnaeus, 1758), Distribution: Adiyaman Host plant: unknown

Blepisanis vittipennis (Reiche, 1877), Distribution: Adiyaman Host plant: unknown

Calamobius filum (Rossi, 1790), Distribution: Gaziantep Host plant: unknown

Calchaenesthes oblongomaculata (Guerin 1844), Distribution: Adiyaman, Gaziantep Host plant: unknown

Callimellum angulatum (Schrank, 1789), Distribution: Adiyaman Host plant: unknown

Cardoria scutellata (Fabricius, 1792), Distribution: Adiyaman Host plant: unknown

Cerambyx dux (Faldermann, 1837), Distribution: Adiyaman, Gaziantep Host plant: Amygdalus communis L.

Cerambyx cerdo Linnaeus, 1758, Distribution: Adıyaman, Şırnak Host plant: unknown

Cerambyx nodulosus Germar, 1817, Distribution: Adıyaman, Mardin Host plant: unknown

Certallum ebulinum (Linnaeus, 1767), Distribution: Adiyaman, Diyarbakır, Gaziantep, Mardin, Şanlıurfa Host plant: unknown

Chlorophorus hungaricus Seidlitz, 1891, Distribution: Gaziantep Host plant: unknown

Chlorophorus sartor (Müller, 1766), Distribution: Gaziantep Host plant: unknown

Chlorophorus varius (Müller, 1766), Distribution: Adıyaman, Diyarbakır, Gaziantep, Mardin, Siirt, Şanlıurfa Host plant: Fruit, Vitis spp.

Chlorophorus varius damascenus (Chevrolat, 1854), Distribution: Diyarbakır Host plant: Prunus persica (L.)

Clytus rhamni Germar, 1817 Distribution: Adiyaman, Gaziantep Host plant: Amygdalus communis L.

Coptosia bithyniensis (Ganglbauer, 1884), Distribution: Adiyaman, Diyarbakır Host plant: unknown

Coptosia compacta (Ménétries, 1832), Distribution: Adiyaman Host plant: unknown

Cortodera colchica Reitter, 1890, Distribution: Adiyaman Host plant: unknown Dorcadion cinerarium (Fabricius, 1787), Distribution: Gaziantep, Şanlıurfa Host plant: unknown 
Dorcadion formosum Kraatz, 1870, Distribution: Gaziantep Host plant: unknown Dorcadion lameeri Théry, 1896, Distribution: Gaziantep Host plant: unknown Dorcadion septemlineatum Waltl, 1838, Distribution: Gaziantep Host plant: unknown

Dorcadion infernale Mulsant \& Rey 1863, Distribution: Diyarbakır Host plant: unknown

Helladia armeniaca Frivaldsky, 1878, Distribution: Adıyaman, Diyarbakır Host plant: unknown

Helladia humeralis (Waltl, 1838), Distribution: Adıyaman, Diyarbakır Host plant: unknown

Helladia millefolii (Adams, 1817), Distribution: Adıaman Host plant: unknown Lampropterus femoratus (Germar, 1824), Distribution: Adiyaman, Diyarbakır, Gaziantep Host plant: unknown

Mallosia herminae Reitter, 1890, Distribution: Şırnak Host plant: unknown

Musaria puncticollis (Faldermann, 1837), Distribution: Diyarbakır Host plant: unknown

Neomusaria balcanica (Frivaldsky, 1835), Distribution: Mardin Host plant: unknown

Neoplagionotus bobelayei (Brullé, 1832), Distribution: Adiyaman, Diyarbakır Host plant: unknown

Oberea oculata (Linnaeus, 1758), Distribution: Adıyaman, Şanlıurfa Host plant: unknown

Opsilia coerulescens (Scopoli, 1763), Distribution: Adıyaman, Diyarbakır Host plant: unknown

Osphranteria coerulescens Redtenbacher, 1850, Distribution: Adiyaman, Diyarbakır, Gaziantep, Mardin, Siirt, Şanlıurfa Host plant: Amygdalus communis L., Malus domestica Borkh., Prunus armeniaca L., Prunus avium L., Vicia sativa L., Prunus persica (L.), Pistacia vera L., Prunus domestica L.,

Oxylia argentata (Ménétriés, 1832), Distribution: Adiyaman, Diyarbakır, Siirt Host plant: unknown

Oxylia languida Mulsant, 1862, Distribution: Siirt Host plant: unknown

Pachytodes erraticus (Dalman, 1817), Distribution: Gaziantep Host plant: unknown

Paraplagionotus floralis (Pallas, 1773), Distribution: Adıaman Host plant: unknown

Phymatodes testaceus (Linnaeus, 1758), Distribution: Adiyaman Host plant: unknown

Phytoecia geniculata Mulsant, 1862, Distribution: Gaziantep Host plant: unknown

Phytoecia puncticollis Faldermann, 1837, Distribution: Diyarbakır Host plant: unknown

Phytoecia virgula (Charpentier, 1825), Distribution: Adiyaman Host plant: unknown

Pilemia annulata (Hampe, 1852), Distribution: Adıyaman Host plant: unknown 
Pilemia hirsutula (Frölich, 1793), Distribution: Adıyaman Host plant: unknown Plagionotus arcuatus (Linnaeus, 1758), Distribution: Diyarbakır Host plant: unknown

Plagionotus floralis (Pallas, 1773), Distribution: Adiyaman Host plant: Trifolium Pseudovadonia livida (Fabricius, 1776), Distribution: Adiyaman, Gaziantep Host plant: unknown

Purpuricenus budensis (Götz, 1783), Distribution: Adıaman, Gaziantep, Siirt Host plant: Amygdalus communis L.

Ropalopus clavipes (Fabricius, 1775), Distribution: Adiyaman Host plant: unknown

Stenopterus kraatzi (Pic, 1892), Distribution: Adiyaman Host plant: unknown

Stenopterus rufus (Linnaeus, 1767), Distribution: Adiyaman, Gaziantep Host plant: unknown

Stenurella bifasciata (O.F. Müller, 1776), Distribution: Gaziantep Host plant: unknown

Stictoleptura cordigera (Füssli, 1775), Distribution: Adıyaman, Gaziantep Host plant: unknown

Stictoleptura tripartita (Heyden, 1889), Distribution: Adiyaman Host plant: unknown

Stromatium unicolor (Olivier, 1795), Distribution: Gaziantep Host plant: unknown

Family Chrysomelidae

Altica deserticola (Weise, 1889), Distribution: Batman Host plant: unknown

Antipus macropus (Illiger, 1800), Distribution: Diyarbakır, Siirt Host plant: Pistacia vera L.

Aphthona atrocaerulea (Stephens, 1831), Distribution: Mardin, Siirt Host plant: unknown

Aphthona franzi Heikertinger, 1944, Distribution: Diyarbakır Host plant: unknown

Aphthona flaviceps Allard, 1859, Distribution: Diyarbakır Host plant: Pistacia vera $\mathrm{L}$.

Aphthona gracilis Fadermann, 1837, Distribution: Diyarbakır Host plant: unknown

Aphthona maculata Allard, 1876, Distribution: Diyarbakır Host plant: Pistacia vera $\mathrm{L}$.

Aphthona semicyanea melanopeza Jacob, 1900-1901, Distribution: Diyarbakır Host plant: unknown

Cassida sp., Distribution: Diyarbakır Host plant: Pistacia vera L.

Cassida brevis Weise, 1884, Distribution: Siirt Host plant: unknown

Cassida linnavuorii Borowiec, 1986, Distribution: Mardin Host plant: unknown

Cassida margaritacea Schaller, 1783, Distribution: Diyarbakır Host plant: unknown

Cassida nobilis Linnaeus, 1758, Distribution: Diyarbakır, Mardin Host plant: Amygdalus communis L. 
Cassida palaestina Reiche, 1858, Distribution: Diyarbakır, Gaziantep Host plant: unknown

Cassida pannonica Suffrian, 1844, Distribution: Diyarbakır Host plant: unknown Cassida prasina Illiger, 1798, Distribution: Gaziantep Host plant: unknown

Cassida saucia Weise, 1889, Distribution: Batman, Gaziantep Host plant: unknown

Cassida subferruginea Schrank, 1776, Distribution: Siirt, Şanlıurfa Host plant: unknown

Cassida viridis Linnaeus, 1758, Distribution: Diyarbakır, Şanlıurfa Host plant: unknown

Chaetocnema montenegrina Heikertinger, 1912, Distribution: Diyarbakır Host plant: unknown

Cheilotoma musciformis (Goeze, 1777), Distribution: Gaziantep Host plant: unknown

Chrysolina polita (Linnaeus, 1758), Distribution: Mardin Host plant: unknown

Chrysolina chalcites (Germar, 1824), Distribution: Diyarbakır Host plant: unknown

Chrysolina sahlbergi (Ménétriés, 1832), Distribution: Diyarbakır Host plant: unknown

Chrysomelina chalcites (Germar, 1824), Distribution: Diyarbakır Host plant: unknown

Cryptocephalus duplicatus Suffrian, 1847, Distribution: Siirt Host plant: unknown

Cryptocephalus moraei (Linnaeus, 1758), Distribution: Diyarbakır Host plant: unknown

Cryptocephalus octacosmus Bedel, 1891, Distribution: Diyarbakır, Mardin Host plant: unknown

Clytra cingulata Weise, 1898, Distribution: Diyarbakır, Gaziantep Host plant: unknown

Clytra bodemeyeri Weise, 1900, Distribution: Gaziantep, Mardin, Siirt Host plant: unknown

Clytra novempunctata Olivier, 1808, Distribution: Gaziantep, Mardin, Siirt Host plant: unknown

Colaphellus sophiae (Schaller, 1783), Distribution: Diyarbakır Host plant: unknown

Coptocephala gebleri Gebler, 1841, Distribution: Diyarbakır Host plant: unknown

Cyaniris limbata Steven, 1806, Distribution: Diyarbakır, Gaziantep, Mardin, Siirt, Şanlıurfa Host plant: unknown

Cyaniris viridana Lacordaire, 1848, Distribution: Southeastern Anatolia Region Host plant: unknown

Entomoscelis adonidis (Pallas, 1771), Distribution: Diyarbakır, Gaziantep Host plant: unknown 
Epitrix hirtipennis (Melsheimer, 1847), Distribution: Diyarbakır Host plant: Pistacia vera $\mathrm{L}$.

Exosome thoracica Rdt., Distribution: Şanlıurfa Host plant: unknown

Galeruca interrupta Illiger, 1802, Distribution: Mardin Host plant: Prunus avium L.

Gastrophysa polygoni (Linnaeus, 1758), Distribution: Diyarbakır Host plant: unknown

Gynandrophtalma limbata Steven, 1806, Distribution: Adıyaman, Diyarbakır, Gaziantep, Mardin, Siirt, Şanlıurfa Host plant: Pistacia vera L.

Gynandrophtalma viridana Lacordaire, 1848, Distribution: Adiyaman, Batman, Diyarbakır, Gaziantep, Mardin, Siirt, Șanlıurfa Host plant: Vitis spp.

Gonioctena fornicata (Brüggemann, 1873), Distribution: Diyarbakır, Mardin Host plant: unknown

Hermaeophaga ruficollis (Lucas, 1849), Distribution: Diyarbakır Host plant: Pistacia vera L.

Hispa atra Linnaeus, 1767, Distribution: Batman, Diyarbakır, Gaziantep, Siirt Host plant: unknown

Labidostomis asiatica Faldermann, 1837, Distribution: Diyarbakır, Gaziantep Host plant: unknown

Labidostomis brevipennis Faldermann, 1837, Distribution: Diyarbakır, Mardin Host plant: Amygdalus communis L.

Labidostomis cyanicornis Germar, 1822, Distribution: Siirt Host plant: unknown

Labidostomis decipiens Faldermann, 1837, Distribution: Şanlıurfa Host plant: unknown

Labidostomis longimana (Linnaeus, 1761), Distribution: Siirt Host plant: Pistacia vera L.

Labidostomis mesopotamica Heyden, 1886, Distribution: Mardin Host plant: unknown

Labidostomis oertzeni Weise, 1889, Distribution: Diyarbakır, Mardin, Siirt Host plant: unknown

Labidostomis propinqua Faldermann, 1837, Distribution: Diyarbakır Host plant: unknown

Lema melanopa Linnaeus, 1758, Distribution: Diyarbakır, Siirt Host plant: unknown

Longitarsus sp., Distribution: Diyarbakır Host plant: Pistacia vera L.

Longitarsus albineus (Foudras, 1860), Distribution: Diyarbakır Host plant: unknown

Longitarsus alfierii Pic 1923, Distribution: Diyarbakır Host plant: Pistacia vera L.

Longitarsus anchusae (Paykull, 1799), Distribution: Diyarbakır Host plant: unknown

Longitarsus dichrous Iablokoff-Khnzorian 1962, Distribution: Diyarbakır Host plant: unknown

Longitarsus fallax Weise, 1888, Distribution: Diyarbakır Host plant: unknown 
Longitarsus ganglbaueri Heikertinger, 1912, Distribution: Diyarbakır Host plant: unknown

Longitarsus lycopi (Foudras, 1860), Distribution: Diyarbakır Host plant: unknown

Longitarsus parvulus (Paykull, 1799), Distribution: Diyarbakır Host plant: unknown

Longitarsus salviae Gruev, 1975, Distribution: Diyarbakır Host plant: Pistacia vera $\mathrm{L}$.

Longitarsus suturellus (Duftschmid, 1825), Distribution: Diyarbakır, Şanlıurfa Host plant: unknown

Longitarsus ochroleucus (Marsham, 1802), Distribution: Diyarbakır Host plant: Pistacia vera L.

Oulema melanopa (Linnaeus, 1758), Distribution: Mardin Host plant: Prunus avium $\mathrm{L}$.

Pachybrachis fimbriolatus (Suffrian, 1848), Distribution: Diyarbakır Host plant: unknown

Phaedon cochleariae (Fabricius, 1792), Distribution: Diyarbakır, Gaziantep Host plant: unknown

Phratora vitellinae (Linnaeus, 1758), Distribution: Diyarbakır, Mardin Host plant: unknown

Phyllotreta corrugata Reiche, 1858, Distribution: Diyarbakır, Gaziantep Host plant: unknown

Phytodecta fornicatus Brüggem, 1873, Distribution: Adiyaman, Batman, Diyarbakır, Gaziantep, Mardin, Siirt, Şanlıurfa, Şırnak Host plant: Amygdalus communis L., Avena sativa L.

Plagiodera versicolora (Laicharting, 1781), Distribution: Diyarbakır, Mardin Host plant: unknown

Prasocuris junci (Brahm, 1790), Distribution: Diyarbakır Host plant: unknown Psylliodes anatolica Gök \& Çilbiroğlu, 2004, Distribution: Diyarbakır Host plant: Pistacia vera L.

Psylliodes hyoscyami (Linnaeus, 1758), Distribution: Diyarbakır Host plant: unknown

Psylliodes sophiae Heikertinger, 1914, Distribution: Diyarbakır Host plant: unknown

Rhaphidopalpa foveicollis Lucas, 1849, Distribution: Adiyaman, Batman, Diyarbakır, Gaziantep, Mardin, Siirt, Şanlıurfa, Şırnak Host plant: Amygdalus communis L., Avena sativa L.

Smaragdina limbata (Steven, 1806), Distribution: Diyarbakır, Mardin Host plant: Amygdalus communis L., Prunus avium L.

Smaragdina scutellaris (Lefevre, 1872), Distribution: Diyarbakır, Mardin Host plant: Amygdalus communis L.

Smaragdina unipunctata (Olivier, 1808), Distribution: Diyarbakır, Gaziantep, Mardin, Şanlıurfa Host plant: Prunus avium L. 
Smaragdina viridana (Lacordaire, 1848), Distribution: Mardin Host plant:

Prunus avium L.

$$
\text { Family Bruchidae }
$$

Acanthoscelides obtectus (Say, 1831), Distribution: Southeastern Anatolia Region Host plant: unknown

Bruchus ervi Frölich, 1799, Distribution: Diyarbakır, Gaziantep, Mardin, Şanlıurfa Host plant: Lens culinaris Medik.

Bruchus dentipes (Baudi, 1886), Distribution: Southeastern Anatolia Region Host plant: unknown

Bruchus lentis Frölich, 1799, Distribution: Gaziantep Host plant: Lens culinaris Medik.

Bruchus nubilus (Boheman, 1833), Distribution: Adıyaman, Batman, Diyarbakır, Gaziantep, Mardin, Siirt, Şanlıurfa Host plant: Legumes and forage crops

Bruchus tetragonus (Baudi, 1886), Distribution: Southeastern Anatolia Region Host plant: unknown

Bruchus tristis Bohemann, 1833, Distribution: Adiyaman, Batman, Diyarbakır, Gaziantep, Mardin, Siirt, Şanlıurfa Host plant: Legumes and forage crops

Bruchus ulicis Mulsant \& Rey, 1858, Distribution: Adiyaman, Batman, Diyarbakır, Gaziantep, Mardin, Siirt, Şanlıurfa Host plant: Legumes and forage crops

Bruchidius albopictus (Allard, 1883), Distribution: Southeastern Anatolia Region Host plant: unknown

Bruchidius anobioides (Baudi, 1886), Distribution: Southeastern Anatolia Region Host plant: unknown

Bruchidius bimaculatus (Olivier, 1795), Distribution: Adiyaman, Batman, Diyarbakır, Gaziantep, Mardin, Siirt, Şanlıurfa Host plant: Legumes and forage crops

Bruchidius fulvus (Allard, 1883), Distribution: Diyarbakır Host plant: Licorice Bruchidius foveolatus (Gyllenhal, 1833), Distribution: Southeastern Anatolia Region Host plant: unknown

Bruchidius trifolii (Motschulsky, 1873), Distribution: Southeastern Anatolia Region Host plant: unknown

Bruchidius quinqueguttatus (Olivier, 1795), Distribution: Adıaman, Batman, Diyarbakır, Gaziantep, Mardin, Siirt, Şanlıurfa Host plant: Legumes and forage crops

Caryedon palestinicus Southgate, 1979, Distribution: Şanlıurfa Host plant: Light trap

Callosobruchus maculatus (Fabricius, 1775), Distribution: Adıyaman, Batman, Diyarbakır, Gaziantep, Mardin, Siirt, Şanlıurfa Host plant: Legumes and forage crops. 
Superfamily Cleroidea

Family Trogossitidae

Tenebroides mauritanicus Linnaeus, 1758, Distribution: Adiyaman, Diyarbakır, Mardin, Siirt, Şanlıurfa Host plant: Amygdalus communis L., the pests of flour, Cucurbita spp.

\section{Superfamily Cucujoidea \\ Family Coccinellidae}

Adalia bipunctata (Linnaeus, 1758), Distribution: Adiyaman, Batman, Diyarbakır, Mardin, Siirt, Şanlıurfa Host plant: Amygdalus communis L., Gossypium hirsitum L., Zea mays L. Host insect: Aphididae, Coccoidea

Adalia decempunctata (Linnaeus, 1758), Distribution: Diyarbakır, Mardin Host plant: Amygdalus communis L. Host insect: Aphididae, Coccoidea

Adalia fasciatopunctata revelierei (Mulsant, 1866), Distribution: Diyarbakır, Mardin Host plant: Unknown Host insect: Aphididae, Coccoidea

Adonia variegata (Goeze, 1777), Distribution: Adiyaman, Batman, Diyarbakır, Gaziantep, Mardin, Siirt, Şanlıurfa, Şırnak Host plant: Zea mays L., Gossypium hirsitum L., Olea europaea L., Pistacia vera L., Vegetables Host insect: Polyphagous, Aphididae, Coccoidea

Chilocorus bipustulatus (Linnaeus, 1758), Distribution: Diyarbakır, Gaziantep, Mardin, Siirt Host plant: Amygdalus communis L., Prunus avium L., Pistacia vera $\mathrm{L}$. Host insect: Coccoidea

Clitostethus arcuatus (Rossi, 1794), Distribution: Diyarbakır Host plant: unknown

Coccinella conglobata Linnaeus, 1758, Distribution: Gaziantep Host plant: Pistacia vera L.

Coccinella septempunctata Linnaeus, 1758, Distribution: Adiyaman, Batman, Diyarbakır, Gaziantep, Mardin, Siirt, Şanlıurfa, Şırnak Host plant: Trifolium, Amygdalus communis L., Prunus avium L., Zea mays L., Gossypium hirsitum L., Olea europaea L., Pistacia vera L., Vegetables Host insect: Polyphagous

Coccinella undecimpunctata Linnaeus, 1758, Distribution: Adiyaman, Batman, Diyarbakır, Gaziantep, Mardin, Siirt, Şanlıurfa Host plant: Trifolium, Amygdalus communis L., Zea mays L., Gossypium hirsitum L., Olea europaea L., Pistacia vera L., Vegetables Host insect: Polyphagous

Coccinula sinuatomarginata (Faldermann, 1837), Distribution: Southeastern Anatolia Region Host plant: unknown

Coccinella quatuordecimpustulata Linnaeus, 1758, Distribution: Southeastern Anatolia Region Host plant: unknown

Exochomus flavipes (Thunberg, 1781), Distribution: Southeastern Anatolia Region Host plant: unknown

Exochomus nigromaculatus (Goeze, 1777), Distribution: Adiyaman, Batman, Diyarbakır, Gaziantep, Mardin, Siirt, Şanlıurfa Host plant: Trifolium, Amygdalus communis L., Pistacia vera L., Vegetables Host insect: Polyphagous 
Exochomus pubescens Küster, 1848, Distribution: Şanlıurfa Host plant: Trifolium,

Adalia fasciatopunctata revelierei Mulsant, 1866, Distribution: Diyarbakır, Mardin Host plant: Pistacia vera L. Host insect: Aphididae, Coccoidea

Exochomus quadripustulatus (Linnaeus, 1758), Distribution: Adiyaman, Batman, Diyarbakır, Gaziantep, Mardin, Siirt, Şanlıurfa Host plant: Amygdalus communis L., Pistacia vera L., Vegetables Host insect: Polyphagous

Harmonia quadripunctata (Pontoppidan, 1763), Distribution: Diyarbakır, Mardin Host plant: Amygdalus communis L. Host insect: Polyphagous

Henosepilachna argus (Geoffroy, 1762), Distribution: Southeastern Anatolia Region Host plant: Unknown Host insect: Polyphagous

Henosepilachna elaterii Rossi, 1794, Distribution: Southeastern Anatolia Region Host plant: Unknown Host insect: Polyphagous

Hippodamia variegata (Goeze, 1777), Distribution: Diyarbakır, Mardin, Şanliurfa Host plant: Amygdalus communis L., Pistacia vera L., Host insect: Polyphagous

Hyperaspis femorata (Motschulsky, 1837), Distribution: Southeastern Anatolia Region Host plant: Unknown Host insect: Polyphagous

Hypraspis quadrimaculata Redtenbacher, 1843, Distribution: Adiyaman, Batman, Diyarbakır, Gaziantep, Mardin, Siirt, Şanlıurfa Host plant: Amygdalus communis L., Cicer arietinum L., Zea mays L., Gossypium hirsitum L., Lens culinaris, Pistacia vera L., Cucumis melo L., Citrillus lanatus L., Host insect: Polyphagous

Hyperaspis repensis (Herbst, 1783), Distribution: Adıyaman, Batman, Diyarbakır, Gaziantep, Mardin, Siirt, Şanlıurfa Host plant: Amygdalus communis L., Pistacia vera L. Host insect: Polyphagous

Hyperaspis polita Weise, 1885, Distribution: Diyarbakır, Şanlıurfa Host plant: Vegetables Host insect: Polyphagous

Myrrha octodecimquttata (Linnaeus, 1758), Distribution: Diyarbakır, Mardin Host plant: Unknown Host insect: Polyphagous

Myzia oblongoquttata (Linnaeus, 1758), Distribution: Southeastern Anatolia Region Host plant: Unknown Host insect: Polyphagous

Nephus (Sidis) caneparii Fürsch \&Uygun, 1980, Distribution: Diyarbakır, Mardin Host plant: Amygdalus communis L. Host insect: Polyphagous

Nephus (Sidis) hiekei (Fursch, 1965), Distribution: Adiyaman, Gaziantep, Mardin, Şanlıurfa Host plant: Olea europaea L. Host insect: Polyphagous

Nephus includens Kirsch, 1870, Distribution: Southeastern Anatolia Region Host plant: Unknown Host insect: Polyphagous

Nephus ludyi Weise, 1879, Distribution: Adiyaman, Batman, Diyarbakır, Gaziantep, Mardin, Siirt, Şanllurfa Host plant: Pistacia vera L. Host insect: Polyphagous

Nephus nigricans Weise, 1879, Distribution: Adıyaman, Batman, Diyarbakır, Gaziantep, Mardin, Siirt, Şanlıurfa Host plant: Amygdalus communis L., Cicer arietinum L., Zea mays L., Gossypium hirsitum L., Lens culinaris L., Cucumis 
melo L., Olea europaea L., Pistacia vera L., Trifolium, Vegetables, Vitis spp., Citrillus lanatus L. Host insect: Polyphagous

Nephus quadrimaculatus (Herbst, 1783), Distribution: Southeastern Anatolia Region Host plant: Unknown Host insect: Polyphagous

Oenopia conglobata (Linnaeus, 1758), Distribution: Adiyaman, Batman, Diyarbakır, Mardin, Siirt, Şanlıurfa Host plant: Amygdalus communis L., Zea mays L., Pistacia vera L., Prunus avium L., Alfalfa Host insect: Polyphagous Oenopia oncina (Olivier, 1808) Distribution: Diyarbakır, Mardin, Siirt Host plant: Amygdalus communis L., Pistacia vera L. Host insect: Polyphagous

Pharoscymnus numidicus (Pic, 1900), Distribution: Diyarbakır, Mardin Host plant: Amygdalus communis L., Pistacia vera L. Host insect: Polyphagous

Pharoscymnus pharoides (Marseul, 1868), Distribution: Adiyaman, Batman, Diyarbakır, Gaziantep, Mardin, Siirt, Şanlıurfa Host plant: Amygdalus communis L., Pistacia vera L. Host insect: Polyphagous

Platynaspis luteorubra (Goeze, 1777), Distribution: Diyarbakır, Mardin, Şanlıurfa Host plant: Amygdalus communis L., Vegetables Host insect: Polyphagous

Propylea quatuordecimpunctata (Linnaeus, 1758), Distribution: Diyarbakır, Şanlıurfa Host plant: Punica granatum L., Vegetables, Host insect: Polyphagous Psyllobora bisoctonotata (Mulsant, 1850), Distribution: Mardin Host plant: Prunus avium L. Host insect: Polyphagous

Psyllobora vigintiduopunctata (Linnaeus, 1758), Distribution: Diyarbakır, Mardin, Siirt, Şanlıurfa Host plant: Amygdalus communis L., Alfalfa Host insect: Polyphagous

Rodolia cardinalis (Mulsant, 1850), Distribution: Southeastern Anatolia Region Host plant: unknown Host insect: unknown

Scymnus apetzi Mulsant, 1846, Distribution: Adiyaman, Batman, Diyarbakır, Gaziantep, Mardin, Siirt, Şanlıurfa Host plant: Gossypium hirsitum L., Olea europaea L., Pistacia vera L. Host insect: Polyphagous

Scymnus (Pullus) araraticus Iablokoff-Khnzorian, 1969, Distribution: Adiyaman, Batman, Diyarbakır, Gaziantep, Mardin, Siirt, Şanlıurfa Host plant: Amygdalus communis L., Zea mays L., Olea europaea L., Punica granatum L., Pistacia vera L., Vegetables Host insect: Polyphagous

Scymnus (Pullus) auritus Thunberg, 1795, Distribution: Diyarbakır, Mardin Host plant: Amygdalus communis L. Host insect: Polyphagous

Scymnus bivulnerus Capra \& Fürsch, 1967, Distribution: Adiyaman, Batman, Diyarbakır, Gaziantep, Mardin, Siirt, Şanlıurfa Host plant: Cucumis melo L., Avena sativa L., Pistacia vera L., Vegetables, Citrillus lanatus L. Host insect: Polyphagous

Scymnus flagellisiphonatus (Fürsch, 1970), Distribution: Adiyaman, Batman, Diyarbakır, Gaziantep, Mardin, Siirt, Şanlıurfa Host plant: Amygdalus communis L., Pistacia vera L., Vegetables Host insect: Polyphagous 
Scymnus flavicollis Redtenbacher, 1843, Distribution: Adiyaman, Batman, Diyarbakır, Mardin, Siirt, Şanlıurfa Host plant: Zea mays L., Gossypium hirsitum L., Vegetables Host insect: Polyphagous

Scymnus frontalis Fabricius, 1787, Distribution: Southeastern Anatolia Region Host plant: Unknown Host insect: Polyphagous

Scymnus inderihensis Mulsant, 1850, Distribution: Diyarbakır, Mardin, Şanlıurfa Host plant: Avena sativa L. Host insect: Polyphagous

Scymnus interruptus (Goeze, 1777), Distribution: Adiyaman, Diyarbakır, Mardin, Siirt, Şanlıurfa Host plant: Gossypium hirsitum L., Vegetables Host insect: Polyphagous

Scymnus levaillantii Mulsant, 1850, Distribution: Adiyaman, Batman, Diyarbakır, Mardin, Siirt, Şanlıurfa Host plant: Zea mays L., Gossypium hirsitum L., Avena sativa L., Punica granatum L. Host insect: Polyphagous

Scymnus marginalis (Rossi, 1794), Distribution: Diyarbakır, Şanlıurfa Host plant: Vegetables, Host insect: Polyphagous

Scymnus mediterraneus Iablokoff-Khnzorian, 1972, Distribution: Diyarbakır, Mardin Host plant: Unknown Host insect: unknown

Scymnus nigrinus Kugelann, 1794, Distribution: Diyarbakır Host plant: Cucumis melo L., Citrillus lanatus L. Host insect: Polyphagous

Scymnus pallipediformis Günther, 1958, Distribution: Adiyaman, Batman, Diyarbakır, Gaziantep, Mardin, Siirt, Şanlıurfa Host plant: Amygdalus communis L., Zea mays L., Cucumis melo L., Pistacia vera L., Punica granatum L., Alfalfa, Vegetables, Vitis spp., Citrillus lanatus L. Host insect: Polyphagous

Scymnus rubromaculatus (Goeze, 1777), Distribution: Adiyaman, Batman, Diyarbakır, Mardin, Şanlıurfa Host plant: Zea mays L., Cucumis melo L., Alfalfa, Punica granatum L., Vegetables, Vitis spp., Citrillus lanatus L. Host insect: Polyphagous

Scymnus suturalis Thunberg, 1795, Distribution: Adıyaman, Batman, Diyarbakır, Gaziantep, Mardin, Siirt, Şanlıurfa Host plant: Pistacia vera L., Host insect: Polyphagous

Scymnus subvillosus (Goeze, 1777), Distribution: Adiyaman, Batman, Diyarbakır, Gaziantep, Mardin, Siirt, Şanlıurfa Host plant: Amygdalus communis L., Zea mays L., Pistacia vera L., Alfalfa, Vegetables, Vitis spp., Prunus avium L. Host insect: Polyphagous

Scymnus syriacus (Marseul, 1868), Distribution: Adiyaman, Batman, Diyarbakır, Gaziantep, Mardin, Siirt, Şanlıurfa Host plant: Pistacia vera L., Prunus avium L. Host insect: Polyphagous

Scymnus quadriguttatus Fursch \& Kreissl 1971, Distribution: Adiyaman, Batman, Diyarbakır, Gaziantep, Mardin, Siirt, Şanlıurfa Host plant: Prunus avium L., Cucumis melo L., Pistacia vera L., Punica granatum L., Vegetables, Vitis spp., Citrillus lanatus L. Host insect: Polyphagous

Scymnus quadrimaculatus (Herbst, 1783) Distribution: Adiyaman, Gaziantep, Mardin, Şanlıurfa Host plant: Olea europaea L. Host insect: Polyphagous 
Coccinella undecimpunctata Linnaeus, 1758 Distribution: Southeastern Anatolia Region Host plant: Unknown Host insect: Polyphagous

Stethorus gilvifrons (Mulsant, 1850), Distribution: Adiyaman, Batman, Diyarbakır, Gaziantep, Mardin, Siirt, Şanlıurfa Host plant: Amygdalus communis L., Zea mays L., Gossypium hirsitum L., Olea europaea L., Pistacia vera L., Alfalfa, Vegetables, Vitis spp., Citrillus lanatus L. Host insect: Polyphagous

Stethorus punctillum (Weise, 1891), Distribution: Adiyaman, Batman, Diyarbakır, Gaziantep, Mardin, Siirt, Şanlıurfa Host plant: Gossypium hirsitum L., Pistacia vera L., Host insect: Polyphagous

Synharmonia conglobata (Linnaeus, 1758), Distribution: Adiyaman, Batman, Diyarbakır, Gaziantep, Mardin, Siirt, Şanlıurfa Host plant: Olea europaea L., Pistacia vera L., Punica granatum L., Alfalfa, Vegetables, Host insect: Polyphagous

Tea vigintiduopunctata (Linnaeus, 1758), Distribution: Southeastern Anatolia Region Host plant: Unknown Host insect: Polyphagous

Vibidia duodecimguttata (Poda, 1761), Distribution: Southeastern Anatolia Region Host plant: Unknown Host insect: Polyphagous.

Family Nitidulidae

Cybocephalus fodori minor (Endrödy-Younga, 1965), Distribution: Mardin, Siirt Host plant: Vitis spp.

Cybocaphalus sp., Distribution: Diyarbakır Host plant: Pistacia vera L.

Family Phalacridae

Olibrus flaviZea mays L.is (Sturm, 1807), Distribution: Diyarbakır, Mardin, Host plant: Amygdalus communis L.

Stilbus apicalis (Melsheimer, 1844), Distribution: Diyarbakır, Mardin, Host plant: Amygdalus communis L.

Family Silvanidae

Oryzaephilus surinamensis (Linnaeus, 1758), Distribution: Adiyaman, Diyarbakır, Mardin, Siirt, Şanlıurfa, Host plant: Amygdalus communis L., Storage pests, the pests of flour, Cucurbita spp.

\section{CONLUSIONS}

The aim of this study is to make contribution to some faunistical records of Coleoptera of the Southeastern Anatolia Region of Turkey. This region has different climatic conditions and contains different habitats. Coleoptera contains both important phytophagous and also zoophagous insects.

\section{REFERENCES}

Akkaya A. 1995. Güneydoğu Anadolu Bölgesinde Baklagil Yem Bitkilerinde Entomolojik Sorunlar ve Çözüm Önerileri. GAP Bölgesindeki Bitki Koruma Sorunları ve Çözüm Önerileri Sempozyumu, Şanlıurfa, S: 304-316. 
Akkaya A, Uygun N. 1996. Diyarbakır ve Şanlıurfa İlleri Yazlık Sebze Ekosistemindeki Insecta Faunası. Türkiye 3. Entomoloji Kongresi Bildirileri, S: 423-430.

Anonymous, 2003, Measuring biodiversity for conservation, The Royal Society, London. S: 65.

Bolu H. 2002. Güneydoğu Anadolu Bölgesi Antepfıstığı Alanlarındaki Böcek ve Akar Faunasının Saptanmas1. Türkiye Entomoloji Dergisi, S: 197-208.

Bolu H. 2004. Güneydoğu Anadolu Bölgesi Antepfistığı Alanlarında Bulunan Avc1 Coccinellidae Türleri, Yayılış Alanları ve Zararlı Agonoscena pistaciae'nın Populasyon Değişimi Üzerine Etkileri. Bitki Koruma Bülteni, 44 (1-4): 69-77.

Bolu H. 2007. Population Dynamics of Lacebugs (Heteroptera: Tingidae) and its Natural Enemies in Amygdalus communis L. Orchards of Turkey. Journal of the Entomological Research Society, 9(1): 33-37.

Bolu H, Beyarslan A, Yıldırım H, Aktürk Z. 2009. Türkiye' den Atanycolus ivanowi (Kokujev, 1898) (Hymenoptera: Braconidae)' nin Kaydedilen İki Yeni Konukçu Türü. Türkiye Entomoloji Dergisi, 33 (4): 279-287.

Bolu H, Beyarslan A, Yıldırım H, Aktürk Z. 2010. Chlorophorus varius damascenus (Chevrolat 1854) (Coleoptera: Cerambycidae), A New Host Record for Iphiaulax impostor (Scopoli 1763) (Hymenoptera: Braconidae) in Turkey. Türkiye Biyolojik Mücadele Dergisi, 1(2): 181-185.

Bolu H, Çınar M, Özgen İ. 2005. Diyarbakır, Elazı̆̆g, Mardin Bademlerinde Zararlı ve Yararlı Böcek Türlerinin Belirlenmesi ve Önemli Türlerin Populasyon Değişimleri Üzerinde Araştırmalar. Tubitak Proje No: TOGTAG-2886. S. 93.

Bolu H, Gençer L, Özgen İ. 2006. Infestation Rates and Natural Enemies of Mercetaspis halli (Green) (Homoptera: Diaspididae) with New Records from Turkey. Journal of the Entomological Research Society, 8(2): 1-5.

Bolu H, Özgen İ, Ayaz T. 2011. GAP İlleri Badem Ağaçlarında Zararlı Böcek Türleri Üzerinde Bir Değerlendirme. Türkiye IV. Bitki Koruma Kongresi Bildirileri, S: 295.

Bolu H, Özgen İ, Bayram A, Çınar M. 2007. Güneydoğu ve Doğu Anadolu Bölgelerinde Antepfıstığı, Badem ve Kiraz Bahçelerindeki Avcı Coccinellidae Türleri, Yayılış Alanları ve Avları. Harran Üni. Ziraat Fakültesi Dergisi, 11 (1-2): 39-47.

Bolu H, Uygun N. 2003. Güneydoğu Anadolu Bölgesi Antepfistıklarında Coccidae Türleri, Yayılış Alanların, Bulaşma Oranları ve Doğal Düşmanlarının Belirlenmesi. Bitki Koruma Bülteni, 43 (1-4): 111-123.

Bolu H, Uygun N. 2005. Suturaspis pistaciae Lindinger (Hem. : Diaspididae) ve doğal düşmanlarının populasyon gelişmesinin belirlenmesi. Bitki Koruma Bülteni, 45 (1-4): 61-78.

Büyük M, Özpınar A. 1999. Diyarbakır İli Kavun ve Karpuz Ekim Alanlarında Zararlı Aphis gosspii Glover (Homoptera: Apihididae) ve Predatörlerinin Populasyon Gelişimi Üzerinde Bir Araştırma. Türkiye IV. Biyolojik Mücadele Kongresi Bildirileri, S: 249-258.

Casale A, Taglianti AV. 1984. Due nuovi Carabidi cavernicoli dell'Anatolia meridionale (Coleoptera, Carabidae). Fragm. Entomol., Roma., 17 (2): 309-329.

Çelik MY. 1981. Gaziantep ve Çevresinde Antep Fıstıklarında Psylloidea'ya Bağlı Önemli Zararlı Türlerin Tanınmaları Yayılışları, Konukçuları, Kısa Biyolojileri ve Doğal Düşmanları Üzerinde Araştırmalar. Araştırma Eserleri Serisi, 51: 1-107.

Çınar M, Çimen İ, Bolu H. 2004. Elazı ğ ve Mardin İlleri Kiraz Ağaçlarında Zararlı Olan Türler, Doğal Düşmanları ve Önemlileri Üzerinde Gözlemler. Türk. entomol. derg., 28 (3): 213-220. 
Dörtbudak N. 1975. Mardin İli Mercimeklerine Arız Olan Bruschus ervi Fröhl.'nin BiyoEkolojisi ve Mücadele Metodları Üzerinde Araştırmalar. Ankara Bölge Zirai Mücadele Araştırma Enstitüsü Müdürlüğü Yayınları Araştırma Eserleri Serisi, 39: 41-46.

Efil L, Bayram A, Ayaz T, Şenal D. 2010. Şanlıurfa İli Akçakale İlçesi Yonca Alanlarındaki Coccinellidae (Coleoptera) Türleri ile Populasyon Değişimleri ve Türkiye İçin Yeni Bir Kayıt, Exochomus pubescens Küster. Bitki Koruma Bülteni, 50 (3): 101-109.

Ergül C, Dörtbudak N, Akülke A. 1972. Doğu ve Güneydoğu Anadolu Bölgesindeki Hububat ve Mamulleri ile Bakliyat Anbar Zararlılarının Yayılışı ve Zararı Üzerinde Araştırmalar. Bitki Koruma Bülteni, 12 (2):129.

Erkılıç L, Mart C, Yiğit A. 1995. Güneydoğu Anadolu Bölgesi Bă̆ Alanlarında Entomolojik Sorunlar ve Çözüm Önerileri. GAP Bölgesindeki Bitki Koruma Sorunları ve Çözüm Önerileri Sempozyumu (Bildiriler), S: 296-304.

Erwin TL. 1982. Tropical forests: their richness in Coleoptera and other arthropod species. Coleopterists Bulletin, 36, 74-75.

Erwin TL. 1991. How many species are there? Revisited Conservation Biology, 5, $1-4$.

Gaston KJ. 1991. The magnitude of global insect species richness. Conservation Biology, 5: 283-296.

Göven MA, Özgür AF. 1990. Güneydoğu Anadolu Bölgesinde Thrips tabaci Lind(Thysanoptera, Thripidae)'nın Populasyonuna Doğal Düşmanların Etkisi. Türkiye II. Biyolojik Mücadele Kongresi Bildirileri, S: 155-164.

Gözüaçık C. 2012. Karacadağ'ın Entomolojik Açıdan Önemi. Karacadağ. Grafik Tasarım. Baskı Uzman matbacılık ve Ciltleme, İstanbul, 463: 330-339.

Günaydın T. 1972. Güneydoğu ve Doğu Anadolu Bölgelerinde Bağ Zararlıları Üzerinde Sürvey Çalışmaları. Zirai Mücadele Araştırma Yıllığı, 6: 42-52.

Günaydın T. 1978. Güneydoğu Anadolu Bölgesinde Antepfistıklarında Zarar Yapan Böcek Türleri, Tanınmaları, Yayılışları ve Ekonomik Önemleri Üzerinde Araştırmalar. S: 106.

Işıkber AA, Özdamar HÜ, Karcı A. 2005. Kahramanmaraş ve Adıyaman İllerinde Depolanmış Buğdaylar Üzerinde Rastlanan Böcek Türleri ve Bulaşma Oranları. KSÜ Fen ve Mühendislik Dergisi, 8 (1): 107-113.

Karaat Ş. 1986. Doğu ve Güneydoğu Anadolu Bölgelerinde Tütün (Nicotianat tabacum L.)'de Zararlı Olan Böcek Türleri, Tanınmaları Yayılış Alanları ve Zararları Üzerinde Araştırmalar. Diyarbakır Zirai Mücadele Araştırma Enstitüsü Araştırma Eserleri Serisi, 4: 26-34.

Karaat Ş, Göven MA, Mart C. 1986. Güneydoğu Anadolu Bölgesinde Bazı Yabancı Otların Zararına Yaşayan Böcek Türleri Üzerinde İlk İncelemeler. Türkiye I. Biyolojik Mücadele Kongresi Bildirileri, S: 186.

Karaat Ş, Göven MA, Mart C. 1986. Güneydoğu Anadolu Bölgesinde Pamuk Ekim Alanlarında Yararlı Türlerin Genel Durumları. Türkiye I. Biyolojik Mücadele Kongresi Bildirileri, S: 162.

Karaat Ş, Göven MA, Mart C. 1987. Güneydoğu Anadolu Bölgesi Pamuk Alanlarında Zararlılar ile Bitki Gelişim Dönemleri Arasındaki İlişkiler. Türkiye I. Entomoloji Kongresi, S: 188-196.

Karaat Ş, Göven MA, Mart C. 1992. Güneydoğu Anadolu Projesi Alanına Giren İllerde Pamuk Alanlarında Zararlılara Karşı Entegre Mücadele Düzeni. Türkiye II. Entomoloji Kongresi Bildirileri, S: 183-192. 
Karaca V, Gözüaçık C, Şimşek Z. 2012. Güneydoğu Anadolu Bölgesi’nde Hububatın Entomolojik Sorunları ve Çözüm Önerileri. Türk Bilimsel Derlemeler Dergisi, 5 (2): 154-159.

Kısmalı Ş, Tezcan S, Turanlı F, Madanlar N. 1995. Chrysomelidae ve Buprestidae (Colepotera) Familyalarına Bağlı Türlerin GAP Bölgesindeki Durumu. GAP Bölgesindeki Bitki Koruma Sorunları ve Çözüm Önerileri Sempozyumu (Bildiriler), S: 139-148.

Küçükkaykı EC, Şirin Ü, Çalışkan H, Şenyüz Y. 2013. Ground beetle (Carabidae: Coleoptera) records from Kaz dağları (Ida mountain). Biological Diversity and Conservation, 6 (2): 142-149.

Lodos N. 1983. Türkiye Faunasına Ait Ekin Kambur Böcekleri, Zabrus (Lain.) (Coleoptera: Carabidae) Cinsinin Yeniden Gözden Getirilmesi. Türkiye Bitki Koruma Dergisi, 7: 51-63.

Lodos N, Önder F, Adıgüzel N, Şimşek Z. 1984. Diyarbakır (Karacadağ) ‘ da Süne’lerin Ovalara Göç Etmeye Başladığı Dönemde, Kışlak Böcek Faunasını Tespit ile Bazı Böcek Türlerinin Kışlak Yerlerinden Çıkış ve Davranışları Üzerinde Araştırmalar. Türkiye Bitki Koruma Dergisi, 8 (1): 45-58.

Lodos N, Önder F, Şimşek Z. 1984. Diyarbakır (Karacadağ)' da Süne (Eurygaster integriceps Put. ) (Heteroptera- Scutelleridae) 'nin Ovalara Göç Ettiği Dönemde Kışlak Böcek Faunasının Tespiti ve Süne ile Diğer Bazı Türlerin Kışlak Yerlerinden Çıkış ve Göç Etme Davranışları Üzerinde Araştırmalar Coleoptera: Chrysomelidae Türleri. Bitki Koruma Bülteni, 24 (3): 113-118.

Lodos N, Önder F, Şimşek Z. 1984. Diyarbakır (Karacadağ)' da Süne (Eurygaster integriceps Put. ) (Heteroptera Scutelleridae)’ nin Ovalara Göç Ettiği Dönemde Kışlak Böcek Faunasının Tespiti ve Süne ile Bazı Türlerin Kışlak Yerlerinden Çıkış ve Göç Etme Davranışları Üzerinde Araştırmalar. Bitki Koruma Bülteni, 24 (2): $75-87$.

Lodos N. 1986. Türkiye Entomolojisi (Genel, Uygulamal1, Faunistik). S: 580.

Maçan S. 1984. Güneydoğu Anadolu Bölgesi'nde Bağlarda Zarar Yapan Böcek Türleri, Önemlilerinin Tanınmaları, Yayılışları ve Ekonomik Önemleri Üzerinde İncelemeler. Tarım Orman ve Köyişleri Bakanlığı, Diyarbakır Bölge Zirai Mücadele Araştırma Enstitüsü Müdürlüğü Araştırma Eserleri, S: 47.

Maçan G. 1986. Güneydoğu Anadolu Bölgesi’nde Bademlerde Zarar Yapan Böcek Türleri, Önemlilerinin Tanınmaları, Yayılışları ve Ekonomik Önemleri Üzerinde Araştırmalar. Tarım ve Köyişleri Bakanlığı Diyarbakır Bölge Zirai Mücadele Araştırma Enstitüsü Müdürlüğü, Araştırma Eserleri Serisi, S: 82.

Maral H. 2012. Diyarbakır, Mardin ve Elazığ İllerinde Tarım ve Tarım dışı Alanlarındaki Ağaçlarda Bulunan Tingidae (Hemiptera) Türleri İle Bunların Parazitoit ve Predatörlerinin Saptanması ve Zararı Önemli Görülen Türlerin Biyolojisi ve Populasyon Gelişiminin Belirlenmesi. Doktora Tezi, S: 171.

Mart C, Karaat Ş. 1990. Güneydoğu Anadolu Bölgesi Antep Fistığ1 Alanlarında Entomolojik Sorunlar. Türkiye 1. Antepfistığı Sempozyumu Bildiriler, 11-12 Eylül, Gaziantep, S: 160.

May RM. 1990. How many species? Philosophical Transactions of the Royal Society B, 330: 292-304.

Moore B, Allard G. 2008. Climate change impacts on forest health. Forest Health and Biosecurity Working Paper FBS/34E. Rome, FAO. P: 45. 
Ölmez S, Ulusoy MR. 2002. Diyarbakır İlinde Aphidoidea Üst Familyasına Bağlı Türlerin Predatörlerinin Saptanması. Türkiye 5. Biyolojik Mücadele Kongresi, S: 237.

Özar Aİ, Yücel A. 1982. Güneydoğu Anadolu Bölgesi’nde Ambarlanan Hububat Ürün Zararlıları Üzerinde Sürvey Çalışmaları. Bitki Koruma Bülteni, 22: 89-98.

Özgen İ, Karsavuran Y. 2005. Siirt İli Antepfıstığı (Pistecia vera) Agroekosisteminde Bulunan Coccinellidae (Coleoptera) Türleri, Yoğunlukları ve Konukçuları Üzerinde Araştırmalar. GAP IV. Tarım Kongresi, 2: 1393-1396.

Özgen İ, Tok S. 2009. Yeni Bir Antepfıstığı Zararlısı Labidostomis longimana (Linneaus, 1758) (Coleoptera: Chrysomelidae). Harran Üniversitesi Ziraat Fakültesi Dergisi, 13 (1): 13-16.

Özpınar A, Yücel A. 2002. Güneydoğu Anadolu Projesi (GAP) alanındaki pamuklarda zararlı ve avcı böcek türlerinin belirlenmesi. Türkiye 5. Biyolojik Mücadele Kongresi, 4-7 Eylül, Erzurum, S: 247.

Öztürk N, Ulusoy M R, Bayhan E. 2005. Doğu Akdeniz Bölgesi Nar Alanlarında Saptanan Zararlılar ve Doğal Düşman Türleri. Türkiye Entomoloji Dergisi, 29 (3): 225-235.

Price P W. 1997. Insect Ecology. John Wiley \& Sons Inc. New York, P: 874.

Ødegaard F. 2000. How many species of arthropods? Erwin's estimate revised. Biological Journal of the Linnean Society, 71: 583-597.

Serel İ. 1978. Güneydoğu Anadolu Bölgesi Çeltiklerinde (Oryza sativa L.) Zarar Yapan Böcekler, Tanınmaları, Yayılışları ve Ekonomik Önemleri Üzerinde Araştırmalar. Diyarbakır Bölge Zirai Mücadele Araştırma Enstitüsü (Yayınlanmamış Uzmanlık Tezi), S: 89.

Stork N. 1997. How many species are there? Biodiversity and Conservation, 2: 215- 232.

Şimşek Z. 1988. Doğu ve Güneydoğu Anadolu Bölgelerinde Mısır ve Darılarda Zararlı Olan Böcek Türleri, Tanınmaları, Yayılışları Alanları ve Zararları Üzerinde Araştırmalar. Araştırma Eserleri Serisi, 6: 1-84.

Simşek A. 2012. Diyarbakır İli Antepfıstığı (Pistacia vera L.) Alanlarındaki Böcek Faunasının Belirlenmesi. Yüksek Lisans Tezi. S: 89.

Türkmen Ş, Göven MA, Akkaya A. 1992. Güneydoğu Anadolu Bölgesinde Mercimek Ekim Alanlarında Bulunan Böcek Türleri ile Önem Durumlarının Tesbiti Üzerinde Çalışmalar. Türkiye II. Entomoloji Kongresi Bildirileri, S: 715-723.

Yanık E, Ünlü L, Yücel A. 2011. Avcı Anthocoris minki 'nin Antepfistığı Bahçelerinde Bulunan Farklı Predatör Türler, Campylomma lindbergi, Deraeocoris pallens ve Oenopia conglobata ile Birlik İçi Avcılığı. Türkiye IV. Bitki Koruma Kongresi Bildirileri, S: 117.

Yücel A. 1988. Güneydoğu Anadolu Bölgesinde Un Fabrikaları ve Un Değirmenlerinde Bulunan Zararlılar ve Zarar Durumları Üzerinde Ön Çalışmalar. Bitki Koruma Bülteni, 28 (1-2): 57.

Yücel A. 1994. Türkiye Faunası için Yeni Bir Tür: Caryedon palestinicus Southgate (Coleoptera: Bruchidae, Pachymerinae). Türkiye Entomoloji Dergisi, 28 (1): 35 39.

Zeren O, Yabaş C. 1992. Gaziantep İlinde Mercimek Bitkisinde Zararlı Olan Ortadoğu Mercimek Tohum Böecği (Bruchus ervi Fröhl.)' ne Karşı İlaç Denemesi. Zirai Mücadele Araştırma Yıllığı, No: 20-21(1985-1986), S: 24. 